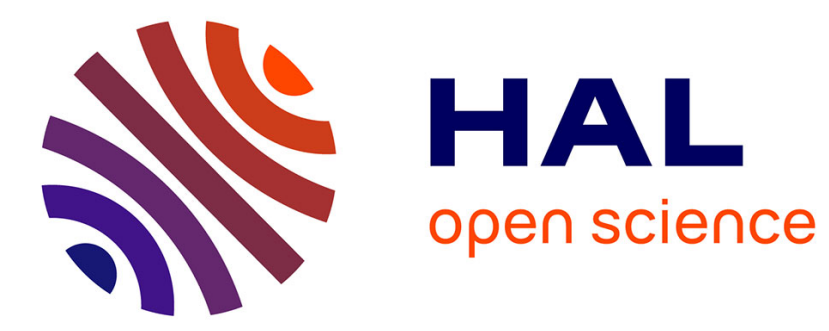

\title{
Road Invariant Extended Kalman Filter for an Enhanced Estimation of GPS Errors using Lane Markings
}

\author{
Zui Tao, Philippe Bonnifait
}

\section{To cite this version:}

Zui Tao, Philippe Bonnifait. Road Invariant Extended Kalman Filter for an Enhanced Estimation of GPS Errors using Lane Markings. IEEE/RSJ International Conference on Intelligent Robots and Systems (IROS 2015), Sep 2015, Hamburg, Germany. pp.3119-3124. hal-01213673

\section{HAL Id: hal-01213673 \\ https://hal.science/hal-01213673}

Submitted on 8 Oct 2015

HAL is a multi-disciplinary open access archive for the deposit and dissemination of scientific research documents, whether they are published or not. The documents may come from teaching and research institutions in France or abroad, or from public or private research centers.
L'archive ouverte pluridisciplinaire HAL, est destinée au dépôt et à la diffusion de documents scientifiques de niveau recherche, publiés ou non, émanant des établissements d'enseignement et de recherche français ou étrangers, des laboratoires publics ou privés. 


\title{
Road Invariant Extended Kalman Filter for an Enhanced Estimation of GPS Errors using Lane Markings
}

\author{
Zui Tao, Philippe Bonnifait
}

\begin{abstract}
Satellite positioning is a key technology for autonomous navigation in outdoors environments. When using standalone computation with mono-frequency receivers, positioning errors are not in accordance with the required performance. Nevertheless, since errors are strongly time-correlated, a GPS fix is quite informative if a shaping model of the positioning errors is carefully handled and made possible by exteroceptive sensors. When driving in a road with a camera detecting lane markings, the lateral error is directly observable by using a lane marking map. It can be well modeled by mixing auto-regressive and random constant models. An algebraic observability study is conducted to prove that this modeling is completely observable in a road frame. A new road invariant Extended Kalman Filter (EKF) is then presented to conserve the observability of every component of the state vector for any road whatever its orientation. The filter manages road changes by using bijective transformations that are detailed. Real experimental results indicate that the performance of the estimation process is significantly improved compared to a classic EKF that is implemented in a fixed working frame with a less informative error modeling.
\end{abstract}

\section{INTRODUCTION}

The ability to estimate the pose of a vehicle with low cost sensors (e.g., [1] [2]) remains a challenging problem for autonomous vehicles. A GPS receiver is useful to compensate Dead-Reckoning's drift and is mandatory for a cold start initialization of the localization system. However, when using L1-GPS positioning with broadcast ephemeris (L1 refers to low cost mono-frequency receivers), errors are not white and can be affected by strong biases, which are mainly due to atmosphere propagation delays and multipath, particularly in urban areas. For this reason, the lane marking aided vehicle localization coupled with GPS information has been studied in many works (see for instance [3][4][5][6]) for autonomous guidance systems. The use of vision sensors (e.g., a camera) simultaneously with an accurate map makes possible to estimate the GPS errors. However, the measurement of a vision sensor is often related to the local geometry of the road and relative to it. In terms of sensor information availability, lateral measurements are more frequent than longitudinal measurements as the firsts ones are done on lane markings and the seconds ones on intermittent features (e.g., stop line). Therefore, the observability of the GPS error along the road axis is weak whereas it is high in the cross-track direction.

In this paper, we present a new way to develop a Kalman filter for this kind of issue. The first idea is to find an efficient model of the GPS errors. The second one is to propose

The authors are with Sorbonne Universités, Université de technologie de Compiègne, CNRS Heudiasyc UMR 7253, CS 60319, 60203 Compiègne cedex

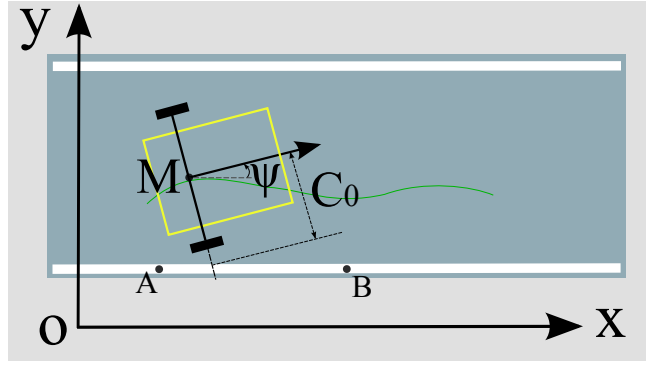

Fig. 1: Road-oriented frame

an adequate working frame to implement a classical EKF. A road invariant EKF algorithm is proposed to handle the enhanced estimation of the GPS errors. This idea is inspired from the Invariant EKF proposed by Bonnabel et al. [7]. Indeed, the localization problem possesses state invariance with respect to road rotations and the observability of every component of the state vector is kept in the road frame. In this work, the observability of the augmented state space is studied in the algebraic framework.

The paper is organized as follows. Section II introduces the system modeling in a road-oriented frame. Section III demonstrates the observability of the proposed modeling. Section IV describes the road invariant EKF algorithm and the localization solver. Outdoor experimental results are presented and analyzed in section $\mathrm{V}$.

\section{SYSTEM MODELING}

The working frame in which a localization solver is implemented plays an important role in terms of error modeling and estimation performance. Often a local ENU (East North Up) frame is used, but as we consider the use of a camera that is able to measure the lateral distance with respect to known lane markings, we propose to consider a modeling of the system in a road-oriented Cartesian frame.

Definition 1: A road-oriented frame is defined to have the same origin as the local ENU frame and its $\mathrm{x}$-axis pointing to the direction of the road in which the vehicle is traveling (cf. Fig. 1).

The localization process uses Dead-Reckoning sensors (yaw rate gyro and wheel speeds) and L1-GPS fixes. As suggested in [8] to manage gyros errors, the GPS error is modelled by the combination of a random constant with an autoregressive process. The random constant handles the bias and the autoregressive process the non-whiteness of the noise.

The state vector is expressed as follows:

$$
X=\left[x, y, \psi, \varepsilon_{\omega}, \varepsilon_{x 1}, \varepsilon_{x 2}, \varepsilon_{y 1}, \varepsilon_{y 2}\right]^{T}
$$


where $(x, y, \psi)$ is the $2 \mathrm{D}$ pose of the vehicle; $\varepsilon_{\omega}$ denotes the gyro bias; $\left(\varepsilon_{x 1}, \varepsilon_{x 2}, \varepsilon_{y 1}, \varepsilon_{y 2}\right)$ are GPS errors on $x$ and $y$ in the road-oriented frame.

The evolution model of the state vector is given by:

$$
\left\{\begin{array}{l}
\dot{x}=v \cdot \cos \psi \\
\dot{y}=v \cdot \sin \psi \\
\dot{\psi}=\omega-\varepsilon_{\omega} \\
\dot{\varepsilon}_{\omega}=0 \\
\dot{\varepsilon}_{x 1}=-\varepsilon_{x 1} / \tau_{1} \\
\dot{\varepsilon}_{x 2}=-\varepsilon_{x 2} / \tau_{2} \\
\dot{\varepsilon}_{y 1}=-\varepsilon_{y 1} / \tau_{1} \\
\dot{\varepsilon}_{y 2}=0
\end{array}\right.
$$

In this model, first order auto-regressive models with time constant $\tau_{1}$ and $\tau_{2}$ are used to model the non-whiteness of the GPS errors [3]. The error in the $x$-direction is split into two components $\left(\varepsilon_{x 1}\right.$ and $\left.\varepsilon_{x 2}\right)$ with different decorrelation time constants in order to manage the frame transformation when the road changes (detailed explanation given in section IV-A). The time constant of $\varepsilon_{y 1}$ is the same as the one of $\varepsilon_{x 1} . v$ is the linear velocity measured by the wheel speed sensors and $\omega$ is the angular velocity measured by the yaw rate gyro.

The last equation of the model associated with $\varepsilon_{y 2}$ plays a important role in our localizer. Indeed, it is a random constant model which is well adapted to estimate the lateral bias of the GPS fix in the road frame.

The exteroceptive sensors that are considered are a GPS receiver and a front-looking camera that detects lane markings. In order to study the structural properties of the modeling with equations easy to handle, let us suppose that the camera and the GPS antenna coincide with point $M$, the origin of the body frame (cf Fig. 1).

Moreover, we consider at this stage that there is only one lane marking, locally represented by a line $[A B]$. The observation model in this case is given by:

$$
C_{0}=\left(y-y_{A}\right) / \cos \psi
$$

$C_{0}$ is the lateral distance measured by the camera in the body frame [9]. $y_{A}$ is the ordinate of point $A$ in the roadoriented frame.

The GPS fixes with their shaping errors are linked to the state by the following model:

$$
\left\{\begin{array}{l}
x_{G P S}=x+\varepsilon_{x 1}+\varepsilon_{x 2} \\
y_{G P S}=y+\varepsilon_{y 1}+\varepsilon_{y 2}
\end{array}\right.
$$

\section{OBSERVABILITY ANALYSIS}

The state space given in the previous section is the basic modeling on which a state observer can be built to estimate the pose of the vehicle and to merge the information from different sensors. The last five components of the state vector with their respective modeling act as shaping filters in the observer. The question that needs to be answered is: Are all the components of the state observable when using the exteroceptive measurements $Y=\left[x_{G P S}, y_{G P S}, C_{0}\right]$ and the proprioceptive inputs $U=[v, w]$ ?

\section{A. Algebraic observability}

We are facing a non-linear system. In such a case, there are two main approaches to study the observability of the state. The most classical one is the local weak observability [10] that relies on the study of a rank condition after linearization and Lie derivatives computation. Another approach relies on the algebraic concept (see [11]).

Theorem 1: (cf. [11]) The state of a system with known internal dynamics is said to be observable if, and only if, there is an algebraic equation linking the state vector to the measured output $Y$ and input $U$ and a finite number of their time derivatives.

Algebraic observability is therefore a different way to study observability. It has also the advantage to provide a analytical way to build a state observer as soon as the derivatives of the inputs and outputs can be estimated with a good quality.

In the following, we study the observability of the different components of the state in the algebraic framework.

\section{B. Observability of vehicle heading $\psi$ and gyro bias $\varepsilon_{\omega}$}

By taking the derivative of Eq. (3), we have:

$$
\dot{y}=\dot{C}_{0} \cdot \cos \psi-\dot{\psi} \cdot C_{0} \cdot \sin \psi
$$

By plugging $\dot{y}=v \cdot \sin \psi$ and $\dot{\psi}=\omega-\varepsilon_{\omega}$ :

$$
v \cdot \sin \psi=\dot{C}_{0} \cdot \cos \psi-\left(\omega-\varepsilon_{\omega}\right) \cdot C_{0} \cdot \sin \psi
$$

By taking the derivative of Eq. (6), we have:

$$
\begin{aligned}
& {\left[\dot{v}+2 \dot{C}_{0}\left(\omega-\varepsilon_{\omega}\right)+\dot{\omega} \cdot C_{0}\right] \sin \psi=} \\
& {\left[\ddot{C}_{0}-C_{0}\left(\omega-\varepsilon_{\omega}\right)^{2}-v\left(\omega-\varepsilon_{\omega}\right)\right] \cos \psi}
\end{aligned}
$$

If $\psi$ is identically null (the vehicle is traveling parallel to the lane marking) then $\dot{\psi}=0$ and we have $\varepsilon_{\omega}=\omega$ (the gyro bias is then observable). Moreover, in this case, we have $\dot{C}_{0}$ that is identically null and so one can observe that $\psi$ is identically null.

Now suppose that $\psi$ is not null. Then we can work out:

$$
\varepsilon_{\omega}=\frac{v \cdot \sin \psi-\dot{C}_{0} \cdot \cos \psi}{C_{0} \cdot \sin \psi}+\omega
$$

$C_{0}$ is physically non null because the lane markings are on the sides of the lane.

By plugging Eq. (8) into Eq. (7), one gets a non-singular expression when the vehicle linear or rotational speeds are not null or when the vehicle accelerates or decelerates:

$$
\begin{aligned}
& \left(\dot{v} \cdot C_{0}-v \cdot \dot{C}_{0}+\dot{\omega} \cdot C_{0}^{2}\right) \sin ^{3} \psi+\left(\ddot{C}_{0} \cdot C_{0}-{\dot{C_{0}}}^{2}\right) \cos ^{3} \psi \\
& +\left(2 \dot{C}_{0}{ }^{2}-\ddot{C}_{0} \cdot C_{0}\right) \cos \psi-v \cdot \dot{C_{0}} \cdot \sin \psi=0
\end{aligned}
$$

From Eq. (9), it is possible to get a function $\Phi_{\psi}$ such as:

$$
\psi=\Phi_{\psi}\left(C_{0}, \dot{C}_{0}, \ddot{C_{0}}, v, \dot{v}, \omega, \dot{\omega}\right)
$$

$\psi$ is therefore observable.

By using Eq. (10) in Eq. (8), a function $\Phi_{\varepsilon_{\omega}}$ can give $\varepsilon_{\omega}$ :

$$
\varepsilon_{\omega}=\Phi_{\varepsilon_{\omega}}\left(C_{0}, \dot{C}_{0}, \ddot{C}_{0}, v, \dot{v}, \omega, \dot{\omega}\right)
$$

which is therefore observable. 
C. Observability of vehicle position $(x, y)$ and of GPS errors

By taking twice the derivative of Eq. (4), we have:

$$
\begin{gathered}
\dot{x}_{G P S}=v \cdot \cos \psi-\varepsilon_{x 1} / \tau_{1}-\varepsilon_{x 2} / \tau_{2} \\
\ddot{x}_{G P S}=\dot{v} \cdot \cos \psi-v \cdot \sin \psi \cdot \dot{\psi}+\varepsilon_{x 1} / \tau_{1}^{2}+\varepsilon_{x 2} / \tau_{2}^{2}
\end{gathered}
$$

Since $\psi$ is observable and as we have a linear system with two unknowns and two equations, we get:

$$
\varepsilon_{x i}=\Phi_{\varepsilon_{x i}}\left(C_{0}, \dot{C}_{0}, \ddot{C}_{0}, \dot{x}_{G P S}, \ddot{x}_{G P S}, v, \dot{v}, \omega, \dot{\omega}\right) i=1,2
$$

So $\varepsilon_{x 1}$ and $\varepsilon_{x 2}$ are observable.

With $x=x_{G P S}-\varepsilon_{x 1}-\varepsilon_{x 2}$ and Eq. (14), $x$ is observable. With $y=y_{A}+C_{0} \cdot \cos \psi$ and Eq. (10), $y$ is observable.

The expression of $\varepsilon_{y 1}$ is given as follows:

$$
\begin{gathered}
\dot{y}_{G P S}=v \cdot \sin \psi-\varepsilon_{y 1} / \tau_{1} \\
\varepsilon_{y 1}=\left(v \cdot \sin \psi-\dot{y}_{G P S}\right) \tau_{1}
\end{gathered}
$$

So $\varepsilon_{y 1}$ is observable.

With $\varepsilon_{y 2}=y_{G P S}-y-\varepsilon_{y 1}$, we can derive that $\varepsilon_{y 2}$ is observable.

So far, we have proved that every element in the state vector $X$ can be expressed by an algebraic function of the components of $Y$ and $U$ and a finite number of their derivatives. We can consequently conclude that the state vector with its associated state space modeling is observable as long as the vehicle moves or accelerates.

\section{ROAD INVARIANT EXTENDED KALMAN FILTER}

The observability of the state has been demonstrated in a road-oriented frame. However, in reality, the orientation of the road changes as the vehicle moves from one road to another. We are now building an EKF that estimates the pose vector of the vehicle from one road to another, in a sequential way.

\section{A. Geometrical transformation}

$R_{O}$ denotes the local ENU frame and $R_{i}$ is the working road-oriented frame, with its $x$-axis pointing to the direction of road $i$. When the vehicle passes from road $i$ to road $j$, the working frame changes from $R_{i}$ to $R_{j}$. Let ${ }^{j} X$ denote the state vector in frame $R_{j}$. The transformation from ${ }^{i} X$ to ${ }^{j} X$ is given by Eq. (17):

$$
\left\{\begin{array}{l}
{ }^{j} x={ }^{i} x \cdot \cos \alpha+{ }^{i} y \cdot \sin \alpha \\
{ }^{j} y=-{ }^{i} x \cdot \sin \alpha+{ }^{i} y \cdot \cos \alpha \\
{ }^{j} \psi={ }^{i} \psi-\alpha \\
{ }^{j} \varepsilon_{\omega}={ }^{i} \varepsilon_{\omega} \\
{ }^{j} \varepsilon_{x 1}={ }^{i} \varepsilon_{x 1} \cdot \cos \alpha+{ }^{i} \varepsilon_{y 1} \cdot \sin \alpha \\
{ }^{j} \varepsilon_{x 2}={ }^{i} \varepsilon_{x 2} \cdot \cos \alpha+{ }^{i} \varepsilon_{y 2} \cdot \sin \alpha \\
{ }^{j} \varepsilon_{y 1}=-{ }^{i} \varepsilon_{x 1} \cdot \sin \alpha+{ }^{i} \varepsilon_{y 1} \cdot \cos \alpha \\
{ }^{j} \varepsilon_{y 2}=-{ }^{i} \varepsilon_{x 2} \cdot \sin \alpha+{ }^{i} \varepsilon_{y 2} \cdot \cos \alpha
\end{array}\right.
$$

Where $\alpha=\theta_{j}-\theta_{i} . \theta_{i}$ and $\theta_{j}$ are respectively the orientation of road $i$ and road $j$ in $R_{O}$ (cf. Fig. 2).

Let ${ }^{i} P$ denote the covariance matrix estimated by the EKF in the working frame $R_{i}$. The transformation from $\left({ }^{i} X,{ }^{i} P\right)$

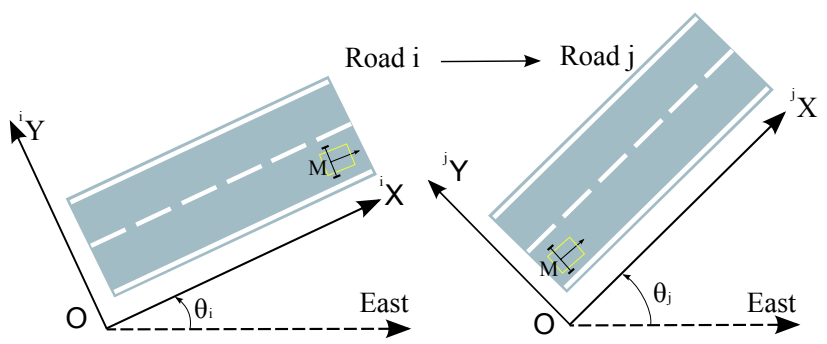

Fig. 2: The vehicle travels from road $i$ to road $j$

to $\left({ }^{j} X,{ }^{j} P\right)$ is described by the function given in Algorithm 1 , where $\operatorname{s} \alpha$ and $\cos$ denote $\sin \alpha$ and $\cos \alpha$ respectively. The road directions being deterministic, this is simply the linear transformation of a random vector.

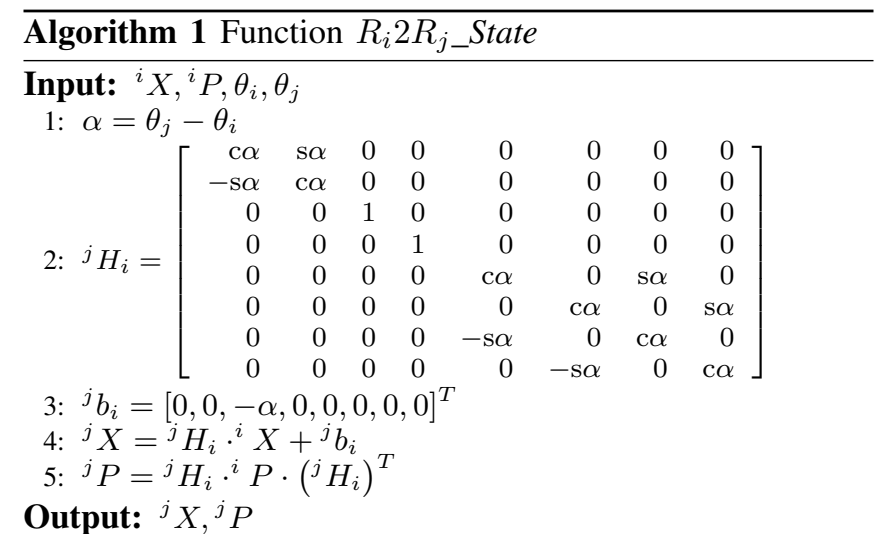

Now, the reason why the bias on ${ }^{i} x$ has been modeled by two components becomes clear. The lateral bias on ${ }^{i} y$ is modeled by an auto-regressive process plus a random constant to get a refined estimation process. If one models the bias on ${ }^{i} x$ by only one component, when the frame changes from $R_{i}$ to $R_{j}$, there is no way to find a bijective transformation. When the vehicle pose is converted from one road frame to another, doing the inverse transformation has to give the same estimate. Mathematically, it means that matrix ${ }^{j} H_{i}$ (cf. Algorithm 1) has to be squared and such that ${ }^{j} H_{i} \cdot{ }^{i} H_{j}=I$ (Identity matrix). It is straightforward to check that our proposal verifies this property.

Since the output of the filter has to be given in the ENU frame $R_{O}$, Algorithm 2 describes the state transformation, where $\left({ }^{O} X,{ }^{O} P\right)$ denotes the Kalman filter estimates expressed in the ENU frame.

\section{B. Road invariant EKF implementation}

The filter is implemented as a discrete EKF triggered by the proprioceptive sensors (typically at a sampling period $T e=0.01 \mathrm{~s}$ ). The GPS and camera measurements are used as soon as they are available. The filter is described in Algorithm 3. ${ }^{i} A$ and ${ }^{i} B$ denote the coordinates of the detected lane marking $[A B]$ in $R_{i}$. 

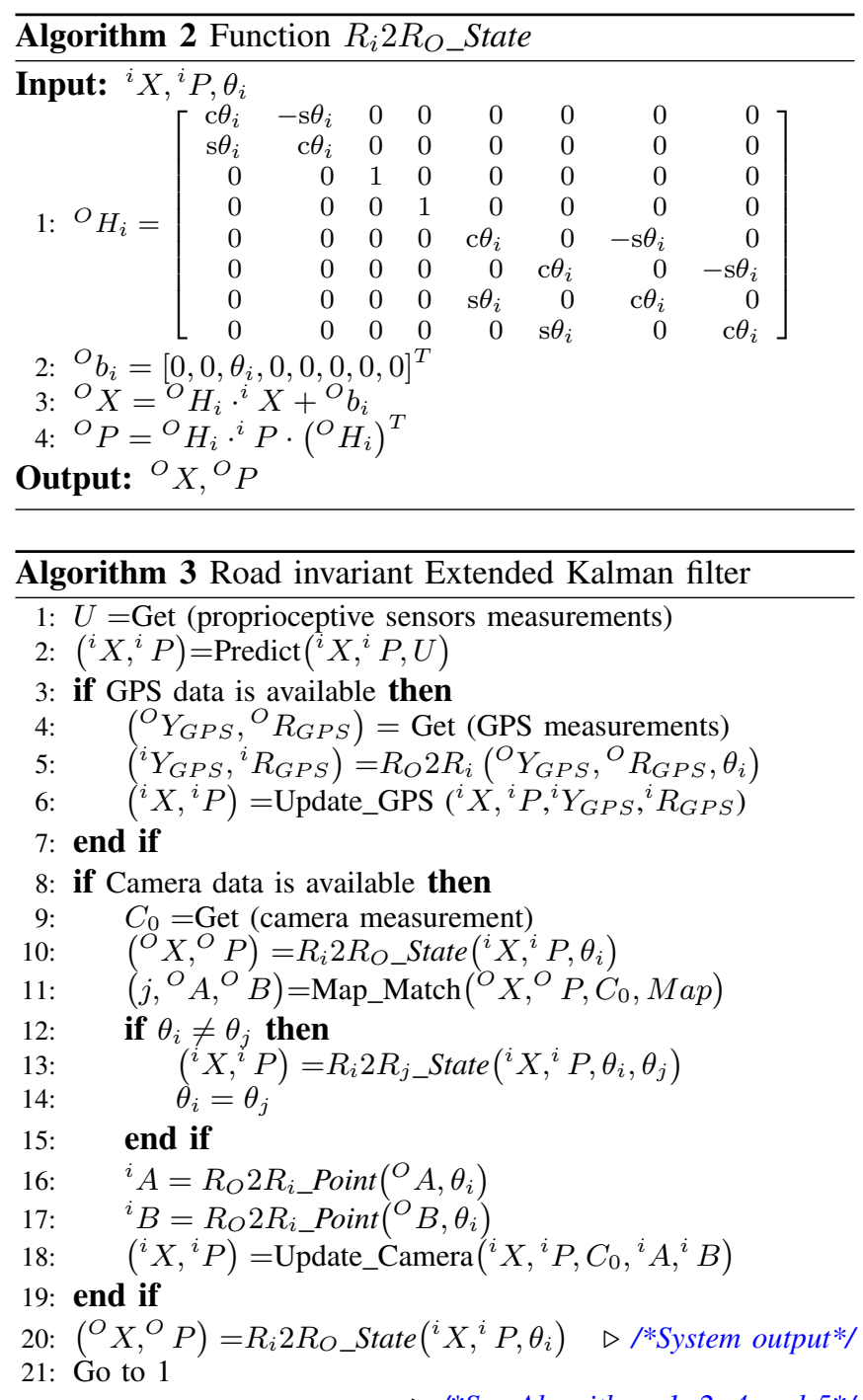

$\triangleright / *$ See Algorithms 1, 2, 4 and 5*/

1) Prediction: When the proprioceptive sensors are available, the function $\operatorname{Predict}\left({ }^{i} X,{ }^{i} P, U\right)$ consists in computing:

$$
{ }^{i} X=f\left({ }^{i} X, U\right) \Longleftrightarrow\left\{\begin{array}{l}
{ }^{i} x={ }^{i} x+T e \cdot v \cdot \cos \left({ }^{i} \psi\right) \\
{ }^{i} y={ }^{i} x+T e \cdot v \cdot \sin \left({ }^{i} \psi\right) \\
{ }^{i} \psi={ }^{i} \psi+T e \cdot\left(\omega-{ }^{i} \varepsilon_{\omega}\right) \\
{ }^{i} \varepsilon_{\omega}={ }^{i} \varepsilon_{\omega} \\
{ }^{i} \varepsilon_{x 1}=a_{1} \cdot{ }^{i} \varepsilon_{x 1} \\
{ }^{i} \varepsilon_{x 2}=a_{2} \cdot{ }^{i} \varepsilon_{x 2} \\
{ }^{i} \varepsilon_{x y 1}=a_{1} \cdot{ }^{i} \varepsilon_{y 1} \\
{ }^{i} \varepsilon_{y y 2}={ }^{i} \varepsilon_{y 2}
\end{array}\right.
$$

and

$$
\left\{\begin{array}{c}
{ }^{i} P=A \cdot{ }^{i} P \cdot A^{T}+B \cdot N \cdot B^{T}+Q \\
A=\frac{\partial f\left({ }^{i} X, U\right)}{\partial^{i} X}, B=\frac{\partial f\left({ }^{i} X, U\right)}{\partial U}
\end{array}\right.
$$

The measurement noises on $v$ and $\omega$ are supposed to be zero-mean independent white noises, $N$ denotes their covariance matrix. $Q$ is the covariance matrix of the process noise. $a_{1}=e^{-T e / \tau_{1}}$ and $a_{2}=e^{-T e / \tau_{2}}$.

2) GPS update: When GPS is available, the measurement vector ${ }^{O} Y_{G P S}=\left({ }^{O} x_{G P S},{ }^{O} y_{G P S}\right)$ in the ENU frame $R_{O}$ is

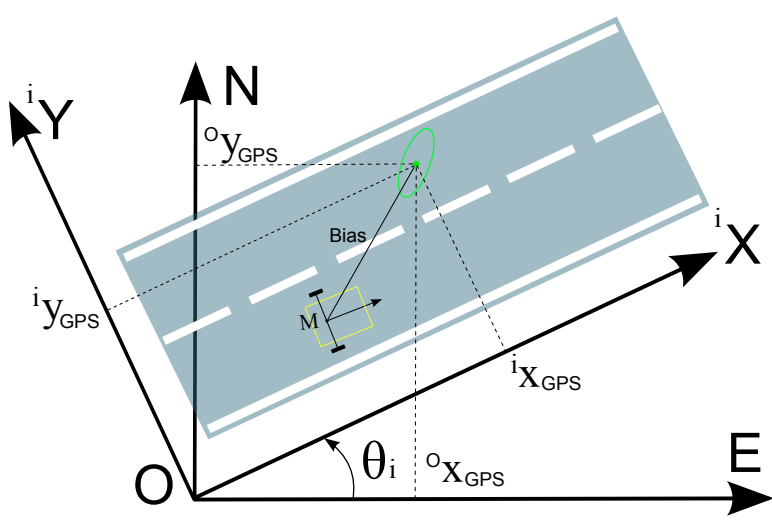

Fig. 3: GPS fix error and frame transformation

transformed into the frame $R_{i}$ (see Fig 3) by Algorithm 4 . The covariance matrix ${ }^{O} R_{G P S}$ given by the receiver is also converted.

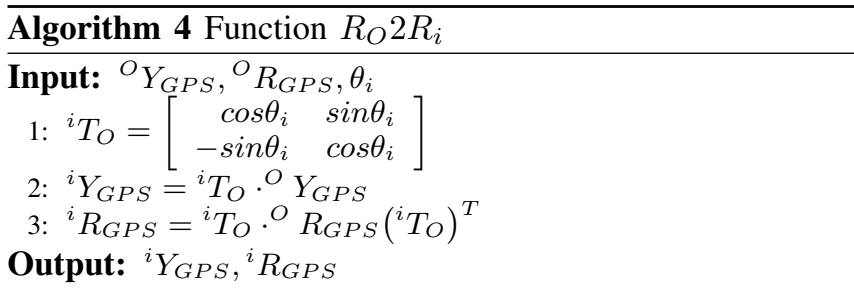

Then, a classical Kalman update step is performed to update $\left({ }^{i} X,{ }^{i} P\right)$ with an innovation gating to reject GPS outliers (e.g., multipath on close buildings). For an accurate data fusion, the level arm of the antenna with respect to the body frame has to be taken into account (see [9] for details).

3) Camera update: In order to update the filter, the map is used as it contains the coordinates of the lane marking. Map matching is therefore done when camera measurements are available. Its goal is to find the road that matches with the detected lane marking. Since the map is defined in $R_{O}$, the first step consists in converting the pose in ENU. Map matching is then applied using the method described in [12]. At this moment, the algorithm checks if the vehicle is in a different road. In this case, the road working frame is modified and the state with its covariance matrix is converted. It just remains to get the coordinates of the lane marking in the road frame which is described by Algorithm 5 and to update the state and covariance matrix by applying an estimation stage of the EKF. In practice, the location of the camera in the body frame is taken into account to get an accurate correction (cf. [12]).

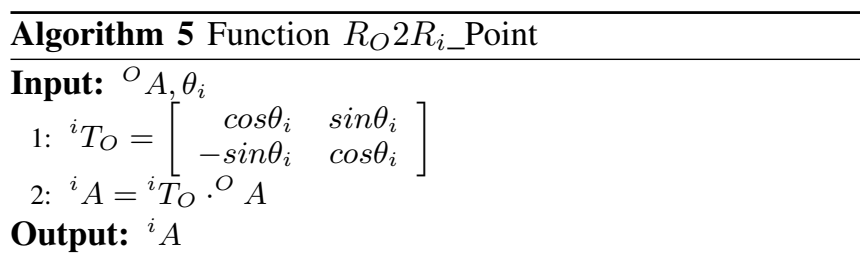




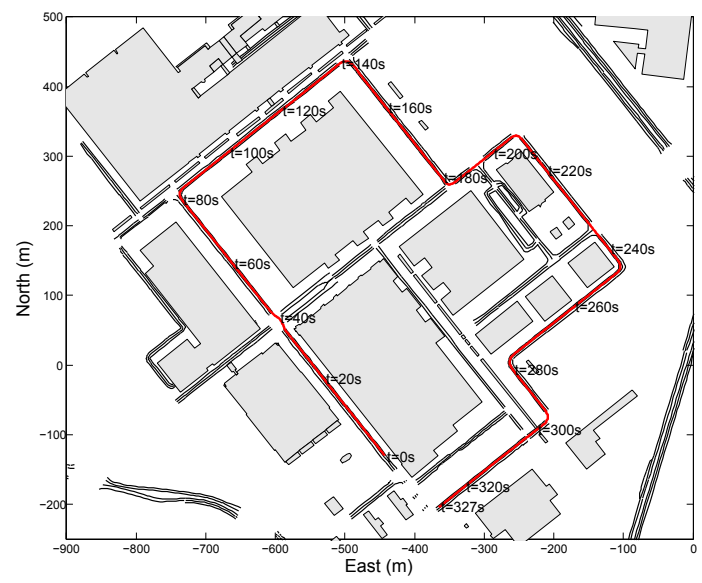

Fig. 4: Test scene and trajectory in the local ENU frame

\section{EXPERIMENTAL RESULTS}

\section{A. Experimental set-up}

In order to evaluate the road invariant Extended Kalman filter, we use real data from outdoor experiments which were carried out near Paris France in May 2013. Three tests were performed on the same road with an experimental automotive vehicle in urban conditions.

The experimental vehicle was equipped with an IMU Oxford RT3000 which provided ground truth data at $100 \mathrm{~Hz}$ rate. A CAN-bus gateway was used to access to the wheel speed sensors and the yaw rate gyro. The measured input $[v, w]$ from the CAN bus was available at $100 \mathrm{~Hz}$. A Mobileye camera was installed behind the windscreen to detect the lane markings at $10 \mathrm{~Hz}$. A low-cost U-blox 6T GPS receiver with a patch antenna was used. It provided position measurements at $5 \mathrm{~Hz}$ with no high-precision GPS measurements.

Fig. 4 shows the test scene in the local ENU frame. The gray bounds represent buildings which are from OpenStreetMap to show the urban conditions of the test scene. The black lines represent the lane marking map expressed by polylines. It mainly consists of two-lane roadways with dashed lane markings in the center of the road and solid ones on both sides of the road. The map has a decimeterlevel accuracy.

The traveling distance for each test was about $2 \mathrm{~km}$ with a typical speed of $30 \mathrm{~km} / \mathrm{h}$. Let take test 1 as an example. The red line stands for the test trajectory (Fig. 4). The vehicle started at $\mathrm{t}=0 \mathrm{~s}$ and stopped at $\mathrm{t}=327 \mathrm{~s}$. Between $\mathrm{t}=80 \mathrm{~s}$ and $t=140 \mathrm{~s}$, the vehicle passed in a strong urban canyon of 300 meters and the U-blox receiver suffered from multipath around $\mathrm{t}=123 \mathrm{~s}$.

\section{B. Localization results}

The method proposed in this paper is compared with a classical loosely coupled EKF implemented in the ENU frame $R_{O}$, in which GPS bias on $x$ and $y$ are modeled using only first order autoregressive processes on the $x$ and $y$ directions. Indeed, it can be shown that adding a random constant model on the two directions makes the system non-observable. As described in Algorithm 3, the output of the solver is a state converted into $R_{O}$. The localization

\begin{tabular}{|l|c|c|c|c|c|c|}
\hline \multirow{2}{*}{} & \multicolumn{3}{|c|}{ Lateral PE (m) } & \multicolumn{3}{c|}{ Longitudinal PE (m) } \\
\cline { 2 - 8 } & I & II & III & I & II & III \\
\hline \hline mean & 1.30 & 0.07 & 0.04 & 1.55 & -0.32 & -0.19 \\
\hline std. dev. & 1.12 & 0.29 & 0.26 & 1.18 & 0.32 & 0.29 \\
\hline median & 0.96 & 0.10 & 0.09 & 1.31 & 0.30 & 0.24 \\
\hline 95th percentile & 3.20 & 0.68 & 0.55 & 3.88 & 0.88 & 0.73 \\
\hline max & 6.78 & 1.83 & 1.37 & 4.69 & 1.50 & 1.36 \\
\hline
\end{tabular}

TABLE I: Error statistics. (I: U-blox; II: ENU EKF; III: road invariant EKF)

\begin{tabular}{|l|c|c|c|}
\hline & median & 95th percentile & $\max$ \\
\hline \hline Lateral Positioning & $10 \%$ & $19 \%$ & $25 \%$ \\
\hline Longitudinal Positioning & $20 \%$ & $17 \%$ & $9 \%$ \\
\hline
\end{tabular}

TABLE II: Improvement by road invariant EKF with respect to ENU EKF

performance is studied in $R_{O}$ by using data replay (both filters have been implemented in $\mathrm{C}++$ to test them with the same framework).

Table I gives the global performance metrics of three tests. U-blox receiver, EKF in the ENU frame (ENU EKF) and road invariant EKF results are reported. Lateral and longitudinal positioning error (PE) are analyzed and compared. One can notice that the localization accuracy is highly improved by using the road invariant EKF with a 95\% lateral PE less than $0.55 \mathrm{~m}$. Table II gives the relative improvement provided by the road invariant EKF with respect to the EKF in the ENU frame, in terms of median, 95th percentile and maximum of the lateral and longitudinal PE. Fig. 5 shows the cumulative distribution of the absolute PE by road invariant EKF and ENU EKF of the three tests. The road invariant EKF gives better estimation on both lateral and longitudinal direction.
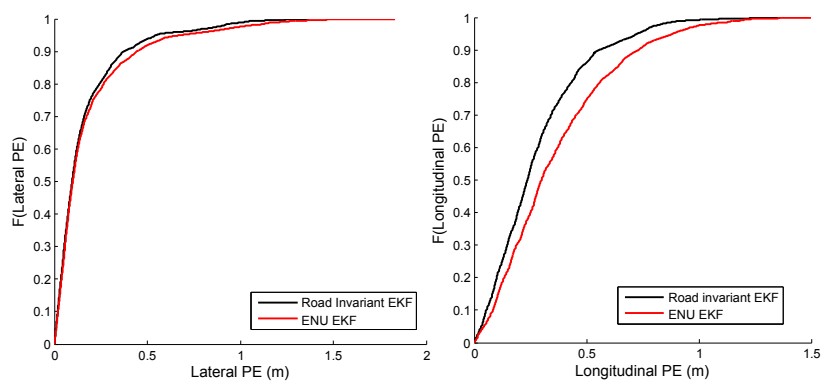

Fig. 5: Cumulative distribution function $(C D F)$ of the positioning errors

Hereafter, result of test 1 is taken to do more analysis. Fig. 6 shows changes of lateral and longitudinal positioning errors over time with $\pm 3 \sigma$ bounds by the road invariant EKF. One can notice that the uncertainty on lateral position increases greatly when the filters loses lane marking measurements. The road invariant EKF remains consistent (99.7\% probability level) with $94.2 \%$ of the data points. The consistence failure rate of ENU EKF is much higher which reaches $24.4 \%$.

\section{Convergence analysis of the GPS errors}

Fig. 7 shows the estimated standard deviation of $\varepsilon_{x 1}$, $\varepsilon_{x 2}, \varepsilon_{y 1}$, and $\varepsilon_{y 2}$ in $R_{O}$. It has to be noticed that they 

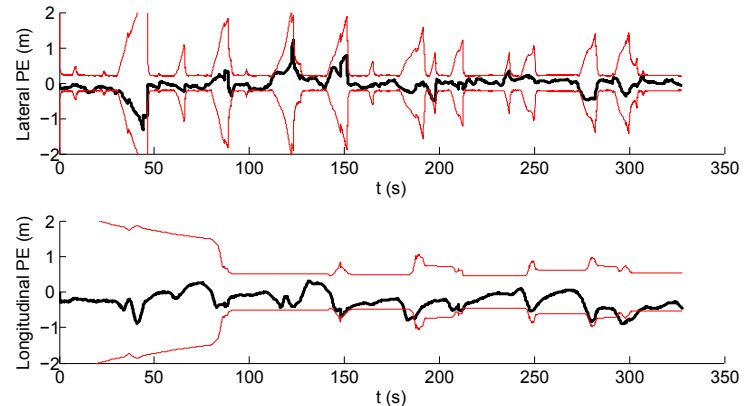

Fig. 6: Lateral and longitudinal PE with road invariant EKF
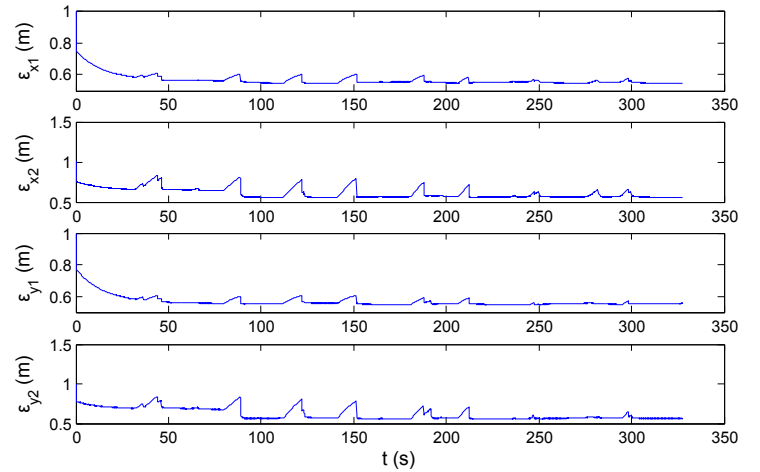

Fig. 7: Estimated standard deviation of the GPS biases

converge towards constants, except when there is no lane marking detection. This is an experimental checking of the observability. By looking at the biases estimates (Fig. 8), one can see that they remain bounded and in the order of magnitude of usual L1-GPS errors.

Figure 9 shows that the sums of the estimate biases in both direction of $R_{O}$ match very well the bias computed with the ground truth equipment.

\section{CONCLUSION}

In this paper, we have proposed a state modeling which handles well the time-correlated errors and bias of L1GPS in a road frame. A road invariant EKF algorithm has been proposed and tested. In particular, the proposed state space model is observable and a bijective transformation between roads guarantees the continuity of the Kalman

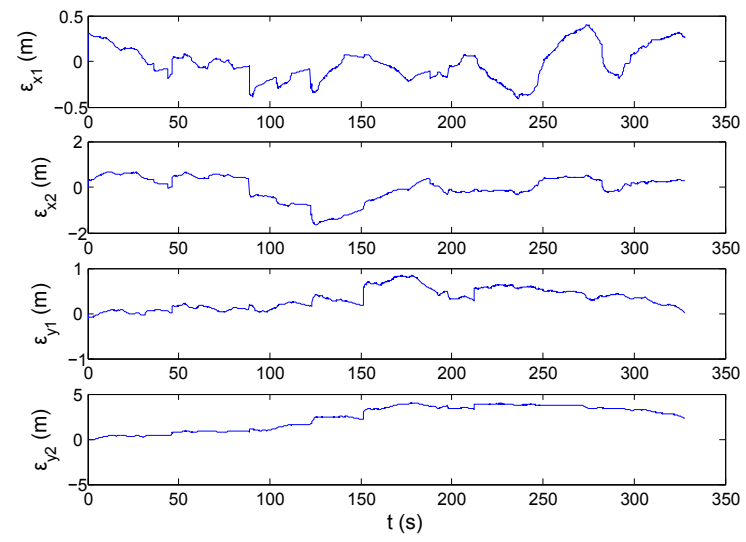

Fig. 8: Estimated biases in $R_{O}$
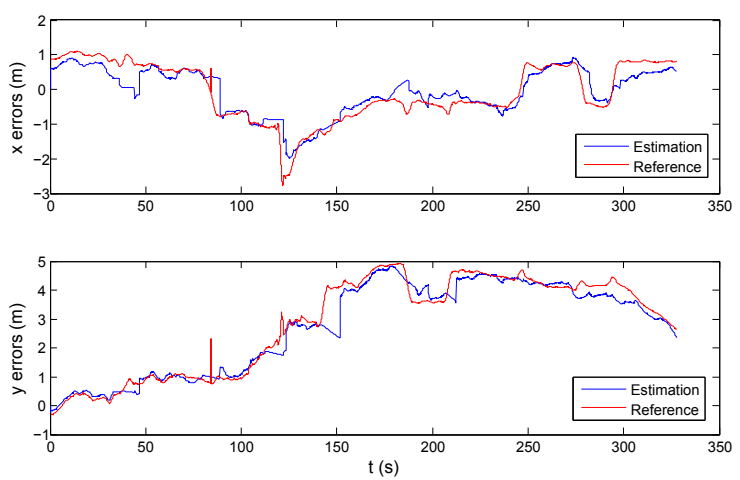

Fig. 9: Estimated biases compared to the reference

filter estimates. The method has been validated with real outdoor data and works much better than a localization solver implemented in the ENU frame in terms of accuracy and consistency because it handles a more refined modeling of the errors. Future work will focus on further validations of the road invariant EKF on roads with different geometries.

\section{Acknowledgments}

The authors would like to thank Vincent Frémont, Stéphane Bonnet and Javier-Ibañez Guzman for their support in the experiments.

\section{REFERENCES}

[1] H. Badino, D. Huber, and T. Kanade, "Real-time topometric localization," in 2012 IEEE International Conference on Robotics and Automation, May 2012, pp. 1635-1642.

[2] M. Brubaker, A. Geiger, and R. Urtasun, "Lost! leveraging the crowd for probabilistic visual self-localization," in 2013 IEEE Conference on Computer Vision and Pattern Recognition, June 2013, pp. 3057-3064.

[3] J. Laneurit, R. Chapuis, and F. Chausse, "Accurate vehicle positioning on a numerical map," International Journal of Control, Automation, and Systems, vol. 3, no. 1, pp. 15-31, March 2006.

[4] I. Miller, M. Campbell, and D. Huttenlocher, "Map-aided localization in sparse global positioning system environments using vision and particle filtering," Journal of Field Robotics, vol. 28, no. 5, pp. 619643, 2011.

[5] K. Jo, K. Chu, and M. Sunwoo, "Gps-bias correction for precise localization of autonomous vehicles," Int. Vehicles Symp., pp. 636641, June 2013.

[6] C. Rose, J. Britt, J. Allen, and D. Bevly, "An integrated vehicle navigation system utilizing lane-detection and lateral position estimation systems in difficult environments for gps," IEEE Transactions on Intelligent Transp. Systems, vol. 15, no. 6, pp. 2615-2629, Dec 2014.

[7] S. Bonnabel, P. Martin, and E. Salaun, "Invariant extended kalman filter: theory and application to a velocity-aided attitude estimation problem," in Proceedings of the 48th IEEE Conference on Decision and Control, Dec 2009, pp. 1297-1304.

[8] Y. Bar-shalom, X.-R. Li, and T. Kirubarajan, Estimation with Applications to Tracking and Navigation. New York, NY, USA: John Wiley \& Sons, Inc., 2002.

[9] Z. Tao, P. Bonnifait, V. Fremont, and J. Ibanez-Guzman, "Mapping and localization using gps, lane markings and proprioceptive sensors," in 2013 IEEE/RSJ International Conference on Intelligent Robots and Systems, Nov 2013, pp. 406-412.

[10] R. Hermann and A. J. Krener, "Nonlinear controllability and observability," IEEE Transactions on Automatic Control, vol. 22, no. 5, pp. 728-740, Oct 1977.

[11] H. Sert, W. Perruquetti, A. Kokosy, X. Jin, and J. Palos, "Localizability of unicycle mobiles robots: An algebraic point of view," IEEE Conf. on Int. Robots and Systems, pp. 223-228, 2012.

[12] Z. Tao and P. Bonnifait, "Tightly coupling gps with lane markings for autonomous vehicle navigation," in 2014 IEEE 17th International Conf. on Intelligent Transp.Systems, Oct 2014, pp. 439-444. 